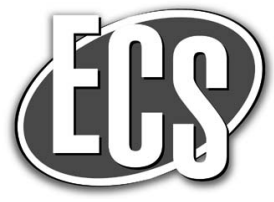

\title{
Dynamic Operation of HT-PEFC: In-Operando Imaging of Phosphoric Acid Profiles and (Re)distribution
}

\author{
S. H. Eberhardt, ${ }^{a}$ M. Toulec, ${ }^{\text {a }}$ F. Marone, ${ }^{\text {b }}$ M. Stampanoni, ${ }^{\text {b,c }}$ F. N. Büchi, ${ }^{\text {a, }}$ \\ and T. J. Schmidt ${ }^{\mathrm{a}, \mathrm{d}, *}$ \\ ${ }^{a}$ Electrochemistry Laboratory, Paul Scherrer Institut, 5232 Villigen PSI, Switzerland \\ ${ }^{b}$ Swiss Light Source, Paul Scherrer Institut, 5232 Villigen PSI, Switzerland \\ ${ }^{c}$ Institute for Biomedical Engineering, ETH Zürich, 8092 Zürich, Switzerland \\ ${ }^{d}$ Laboratory of Physical Chemistry, ETH Zürich, 8093 Zürich, Switzerland
}

\begin{abstract}
Synchrotron based X-ray tomographic microscopy (XTM) is used for imaging and quantifying the redistribution of phosphoric acid (PA) in high temperature polymer electrolyte fuel cells (HT-PEFC) in-operando. The main focus of this work is the redistribution of phosphoric acid under dynamic load conditions. Therefore, two different load cycling protocols were applied and the transient redistribution within the fuel cell components was imaged. XTM, for the first time, revealed that the examined PBI based membrane system exhibits extensive electrolyte migration from cathode to anode under high current density operation. PA flooding of anode gas diffusion layer (GDL) and flow field channels occurred. Implications for technical applications and fuel cell degradation are discussed. Quantification of the migrated electrolyte is made by correlating in-operando grayscale values to ex-situ reference samples. (C) The Author(s) 2015. Published by ECS. This is an open access article distributed under the terms of the Creative Commons Attribution 4.0 License (CC BY, http://creativecommons.org/licenses/by/4.0/), which permits unrestricted reuse of the work in any medium, provided the original work is properly cited. [DOI: 10.1149/2.0751503jes] All rights reserved.
\end{abstract}

Manuscript submitted November 17, 2014; revised manuscript received December 26, 2014. Published January 6, 2015.

High temperature polymer electrolyte fuel cells (HT-PEFC) are operating at temperatures up to $200^{\circ} \mathrm{C}$ using phosphoric acid (PA) doped polybenzimidazole (PBI) based membranes. This fuel cell technology has long been in the focus of research ${ }^{1}$ due to advantages based on the higher operation temperature as compared to standard PEFCs, operating between $60-90^{\circ} \mathrm{C}$. At the higher operating temperatures, the tolerance to fuel gas impurities increases significantly and operation with $\mathrm{CO}$ levels up to $3 \%$ and $\mathrm{H}_{2} \mathrm{~S}$ up to $10 \mathrm{ppm}$ can be achieved. ${ }^{2}$ This renders HT-PEFC especially suitable for stationary combined heat and power (CHP) applications, where a fuel processing unit can easily be thermally integrated and used for reforming hydrocarbon-based fuels without the need of additional gas clean-up. The advantageous characteristics of HT-PEFCs are also determined by the physico-chemical properties of the phosphoric acid electrolyte. First of all, PA has a low vapor pressure at these operating temperatures. This principally allows for long term operation without major electrolyte loss, an important aspect also specifically defined in the US Department of Energy's 2015 targets $^{3}$ (50'000 operating hours for stationary CHP systems). Phosphoric acid also exhibits excellent proton conductivity owing to a fast proton hopping mechanism. ${ }^{4}$ Due to this inherent difference to PFSA-type membranes (water assisted shuttle mechanism), additional humidification is not necessary, which significantly reduces the complexity of HT-PEFC systems. However, only ca. 2 PA molecules per $\mathrm{PBI}$ repeating unit (PA/PBI) are directly interacting with the basic pyridinic nitrogen of PBI. ${ }^{5}$ However, PA doping levels are typically significantly higher, e.g., 5-10 for PA imbibed PBI films ${ }^{6}$ and 20-40 for PBI membranes produced through the so-called poly-phosphoric acid (PPA) process. ${ }^{7}$ It can, therefore, be expected that the bulk of this phosphoric acid is more or less mobile within the molecular pores of the membrane. Hence, movement and redistribution of PA within the porous components (membrane, catalyst layer, micro-porous and gas diffusion layers) of the cell are expected to play a significant role for fuel cell performance and durability. A process which is well known from earlier work on phosphoric acid fuel cells (PAFC), where PA imbibed in a porous silicon carbide separator remains mobile. Also for HT-PEFC several studies have been conducted aiming at understanding electrolyte redistribution from gas diffusion electrode (GDE) to membrane ${ }^{8}$ or membrane electrode assembly (MEA) to porous bipolar plates ${ }^{9}$ but no mechanistic interpretation of the results has been be given yet. This is partially due to the fact, that post mortem analysis of the components cannot describe the PA distribution under operation

${ }^{*}$ Electrochemical Society Active Member.

${ }^{\mathrm{z} E}$-mail: felix.buechi@psi.ch due to diffusion and concentration changes after shutdown of the fuel cell. In-operando measurements targeting the electrolyte distribution are currently limited to electrochemical impedance spectroscopy (EIS) and imaging. While EIS is sensitive to changing proton conductivity, ${ }^{10}$ the interpretation of spectra from technical cells is error prone and no information about PA quantity or location can be derived. X-ray imaging on the other hand has been proven to give valuable information on the water distribution of low temperature polymer electrolyte fuel cells ${ }^{11-13}$ and first results using in-operando X-ray radiography of HT-PEFC ${ }^{13-15}$ indicated the possibility to track changes in PA concentration and distribution. Recently, neutron radiography was also evaluated for imaging PA in HT-PEFC and it was demonstrated to produce sufficient contrast between PA and the structural components to be used as diagnostic tools for these systems. ${ }^{16}$

In this work, synchrotron based X-ray tomographic microscopy will be applied, for the first time, to image in-operando HT-PEFC. The experiments are specifically targeted at imaging the transient phosphoric acid electrolyte redistribution under dynamic load conditions to identify possible fuel cell degradation with respect to electrolyte loss from the electrochemical active area. Additionally, PA concentration is determined in-operando by calibration of grayscale values in the tomographic images. ${ }^{17}$

\section{Experimental}

General.- All experiments were carried out with BASF Celtec membrane electrode assemblies (MEA). These MEA consist of a $\mathrm{H}_{3} \mathrm{PO}_{4}$ doped polybenzimidazole (PBI) membrane with an acid loading of 34-36 $\mathrm{mg}_{\mathrm{H} 3 \mathrm{PO} 4} \mathrm{~cm}^{-2}$ and a thickness of approximately $100 \mu \mathrm{m}$ as shown in Table I. Phosphoric acid itself consists of a mixture of orthophosphoric acid $\mathrm{H}_{3} \mathrm{PO}_{4}, \mathrm{H}_{2} \mathrm{O}$ and to some extent also polyphosphoric acid. The ratio is determined by temperature and water partial pressure. For the sake of simplicity PA is subsequently always referred to as a mixture of $\mathrm{H}_{3} \mathrm{PO}_{4} / \mathrm{H}_{2} \mathrm{O}$ and is quantified by wt $\%$. The

Table I. Membrane electrode assembly material properties (thickness and compression was determined in-operando using XTM).

\begin{tabular}{cccc} 
Component & Material & Loading & Thickness $[\mu \mathrm{m}]$ \\
\hline GDL/MPL & SGL 38 carbon paper & - & 240 \\
Catalyst Layers & Pt/Vulcan XC-72 & $1 \mathrm{mg}_{\mathrm{Pt}} \mathrm{cm}^{-2}$ & 80 \\
Membrane & $\mathrm{PBI} / \mathrm{H}_{3} \mathrm{PO}_{4}$ & $34-36 \mathrm{mg}_{\mathrm{PA}} \mathrm{cm}^{-2}$ & 100
\end{tabular}


Table II. Operating parameters and measurement sequences.

Slow cycling

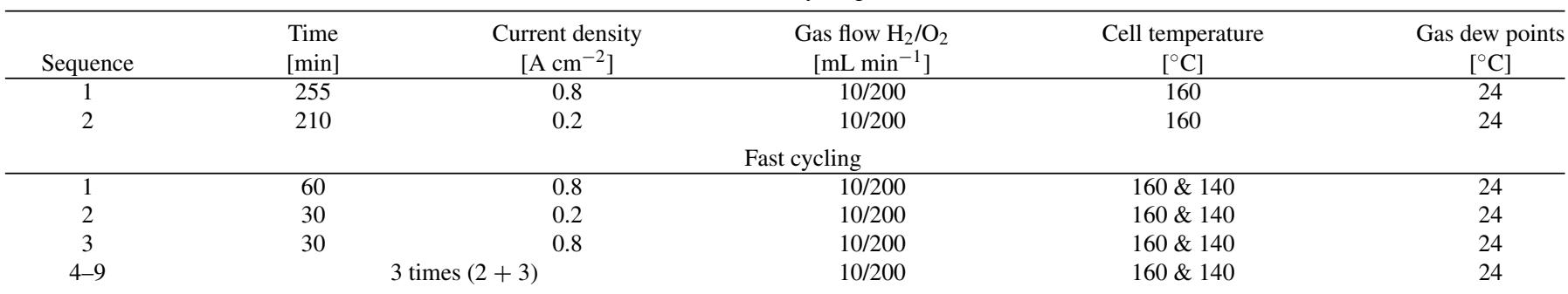

electrodes consist of $\mathrm{Pt} / \mathrm{Vulcan} \mathrm{XC}-72$ supported platinum catalyst with a loading of $1 \mathrm{mg}_{\mathrm{Pt}} \mathrm{cm}^{-2}$ on anode and cathode, respectively, coated onto SGL 38 carbon paper gas diffusion layers (GDL) including a micro-porous layer (MPL).

A specially designed cell, used for imaging low temperature PEFC (LT-PEFC) $)^{18}$ was redesigned to accommodate the specific operation environment of HT-PEFCs. It has a circular active area of $0.2 \mathrm{~cm}^{2}$ and a flow field design with three parallel channels (width $\times$ depth of 0.5 $\times 0.3 \mathrm{~mm}^{2}$ ). MEA compression was adjusted by constant gap using two $320 \mu \mathrm{m}$ PFA sealing gaskets. The resulting GDL compression was $40 \%$.

Two different protocols were used for imaging: (i) slow cycling with current densities changing between 0.2 and $0.8 \mathrm{~A} \mathrm{~cm}^{-2}$ approx. every 4 hours. (ii) fast cycling with load changes every $30 \mathrm{~min}$. The slow cycling protocol was carried out at $160^{\circ} \mathrm{C}$, the fast cycling protocol at 160 and $140^{\circ} \mathrm{C}$. The cell for the slow cycling protocol was conditioned for $4 \mathrm{~h}$ before the first current density step and for the fast cycling protocol a $25 \mathrm{~h}$ conditioning period was applied. Gas flow rates were set to $10 \mathrm{~mL} \mathrm{~min}{ }^{-1} \mathrm{H}_{2}$ and $200 \mathrm{~mL} \mathrm{~min}^{-1} \mathrm{O}_{2}$ (differential operating conditions with minimal gradients along the channels). To prevent membrane drying and shrinking, both gases were humidified at room temperature. Operating conditions for both protocols are summarized in Table II.

$X$-ray imaging.- X-ray tomographic microscopy (XTM) imaging was performed at the TOMCAT (TOmographic Microscopy and Coherent rAdiology experimenTs) beamline of the Swiss Light Source. ${ }^{19}$ At a beam energy of $20 \mathrm{keV}$ a total of 2001 projections were acquired during $180^{\circ}$ sample rotation $\left(0.09^{\circ}\right.$ step angle) in absorption contrast mode with an exposure time of $11 \mathrm{~ms}$ per projection (22 $\mathrm{s}$ acquisition time for a tomographic scan). The cell was positioned as close as possible to the scintillator $(10 \mathrm{~mm})$ and projections were reconstructed using an efficient algorithm based on a Fourier method. ${ }^{20}$ The sCMOS camera (PCO.edge) had a chip size of $2560 \times 2160$ pixels and the resulting voxel size in the tomographic images was $2.34 \mu \mathrm{m}$. All tomograms were flat- and darkfield corrected. The reconstructed slices were saved in 16-bit TIFF format using constant minimum and maximum grayscale level values for all scans.

Image processing. - To obtain quantitative information, the grayscale images need to be segmented into the relevant phases. An overview on the methodology for quantifying all phases in HT-PEFC has been published recently. ${ }^{17}$ For the present data segmentation was performed for the anode GDL and flow field using the open source software Fiji (ImageJ) as well as MATLAB. The image processing workflow is presented here briefly. First a median filter (2D, 3px) was applied, and then a grayscale threshold segmentation window was applied once to obtain the carbon fibers/binder structure of the GDL and once to obtain the phosphoric acid phase. After thresholding an additional 3D cleaning in MATLAB (area opening; cluster size $\leq 6000$ px) of the binary images was implemented to reduce noise due to small, unconnected segmentation artifacts. A sensitivity analysis for determined PA volume was performed and indicates a maximum error $-4 \%$ up to $+11 \%$ for a threshold variation of $\pm 2 \%$. The two final binary datasets consist on the one hand of the pure PA phases and on the other hand of the carbon (fibers/binder) structure. The former is used for PA quantification and the latter for porosity calculations of the empty GDL structure.

Phosphoric acid quantification. - In 3D grayscale images different phases and materials are represented by different grayscale values. The catalyst layer has the highest grayscale value due to the presence of highly absorbing platinum nanoparticles. The phosphoric acid electrolyte has a medium value, followed by the carbon fibers, PBI and void with the lowest gray value. The grayscale of the membrane is therefore mainly dominated by PA. Absorption contrast based reconstruction of the tomographic images gives the possibility to establish a linear relation between grayscale value and a material dependent linear attenuation coefficient $\mu\left(\mathrm{cm}^{-1}\right)$. Therefore, a calibration experiment was performed where MEAs doped with 40, 60, 85 and $100 \mathrm{wt} \% \mathrm{H}_{3} \mathrm{PO}_{4}$ were imaged at room temperature. In these images the grayscale value of the phosphoric acid phase (without carbon) was analyzed. Based on this information and a correction for the temperature dependent PA density the composition of the PA electrolyte in the in-operando experiment can be determined.

\section{Results and Discussion}

This section is divided into a presentation of the imaging results of electrolyte redistribution for two current cycling protocols followed by a quantification of redistributed PA. The results are finally concluded by a discussion with respect to fuel cell performance and degradation.

$P A$ redistribution.- Phosphoric acid distribution in the MEA is expected to be influenced by various parameters, such as cell temperature, gas dew points and in particular also by current density. Current density influences the water vapor balance in the cell and can thus also influence the ion mobilities, in particular the transport number of the proton in the membrane. The influence of current density is investigated using two different protocols. With the slow protocol a single current transient from 0.2 to $0.8 \mathrm{~A} \mathrm{~cm}^{-2}$ and back to $0.2 \mathrm{~A} \mathrm{~cm}^{-2}$ is performed at $160^{\circ} \mathrm{C}$, while with a fast protocol multiple cycles between the same current densities at 160 and $140^{\circ} \mathrm{C}$ are performed. Experimental details are listed in Table II.

At start of break-in of a new MEA, initial PA content in the GDLs originates from the membrane. It was transferred there during MEA preparation, which includes mechanical compression for application of the GDL based electrodes. During break-in at $0.2 \mathrm{~A} \mathrm{~cm}^{-2}$ these PA phases are reduced and at the anode only a few PA filled pores remain in the GDL while at the cathode no segmented PA phase can be observed anymore. However, an increased grayscale value of the cathode GDL (as compared to pure carbon) indicates that small amounts of PA are still present, possibly accumulating within the micro-pores of the carbonaceous binder ${ }^{17}$ and as thin film wetting the fibers. Analysis of imaging results is therefore focused on the anode GDL. A cross-section through the cell is shown in Figure 1. Flow field gas diffusion layer and membrane electrode assembly can clearly be 


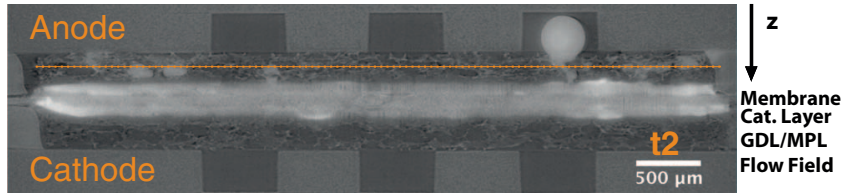

Figure 1. Vertical slice through the MEA illustrating the flow field, GDL/ MPL, catalyst layer and membrane (white: Pt; bright gray: PA; light gray: carbon fibers; dark gray: empty pores). Orange line in anode GDL denotes plane for slices in Figure 2.

identified by the different grayscales. In the anode GDL and flow field channels phosphoric acid can well be observed.

First the slow cycling protocol is analyzed and a comparison of horizontal grayscale slices of the anode GDL at different times during the current density cycle is shown in Figure 2, while a detailed, time resolved volumetric quantification of the PA in GDL and flow field channels is given in Figure 3. When the current is increased from 0.2 to $0.8 \mathrm{~A} \mathrm{~cm}^{-2}$, an almost immediate flow of PA into the anode GDL can be observed as illustrated in Figure $2 t 2$, and the inset in Figure 3. Within 15 min, a continuous path of PA has formed from the membrane to the anode flow field channels leading to a rise in PA volume in the flow field channels and accumulation under the flow field ribs as shown in Figure 3 and Figure 4 (here for two of the droplets the continuous PA path is well visible, for the other droplets it is out of plane). PA saturation in the GDL increases until it reaches a steady-state after approximately 3 hours at $0.8 \mathrm{~A} \mathrm{~cm}^{-2}$ (Figure $2 \mathrm{t} 3$ ). After $4 \mathrm{~h}$ at high current density also the PA in the flow field reaches a steady state and the total PA volume in GDL and flow field adds up to $45 \cdot 10^{-3} \mathrm{~mm}^{3} \mathrm{~mm}^{-2}$ compared to $1.9 \cdot 10^{-3} \mathrm{~mm}^{3}$ $\mathrm{mm}^{-2}$ at low current density. At this point one of the three parallel flow field channels is just about filled with electrolyte.

After 4 hours the current density is decreased back to $0.2 \mathrm{~A} \mathrm{~cm}^{-2}$ and a sudden drop in GDL saturation can be observed concomitant

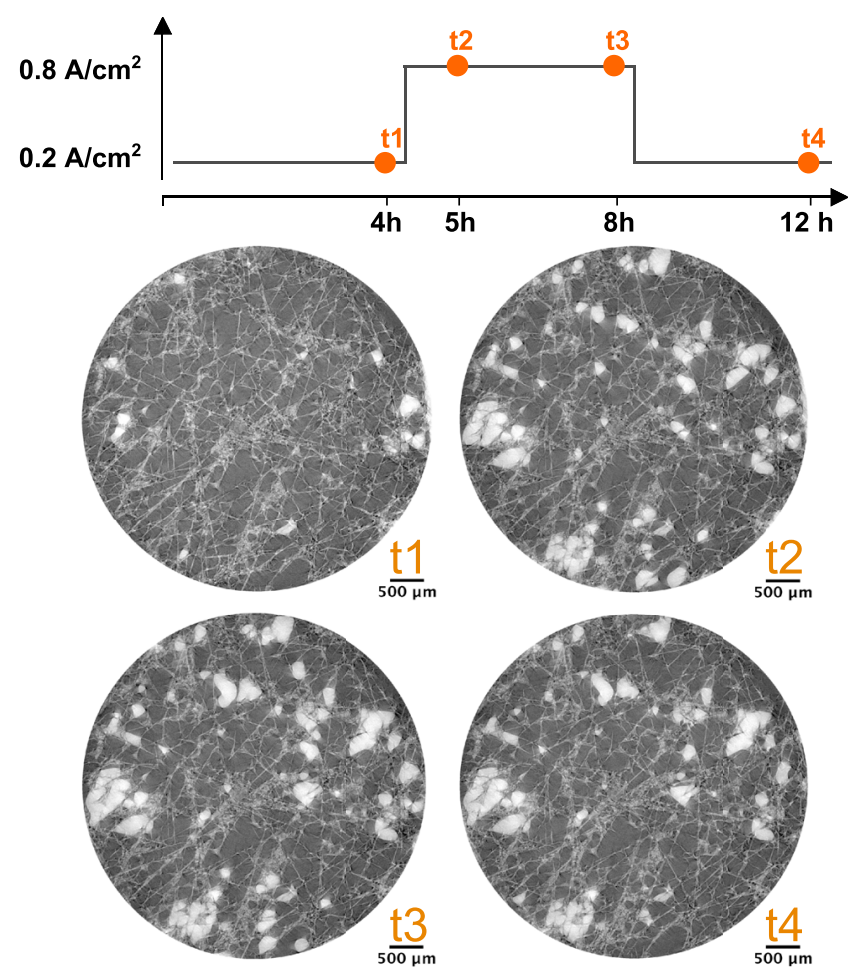

Figure 2. Horizontal slices of the anode GDL in the slow cycling experiment as a function of time (bright gray: PA; light gray: carbon fibers; dark gray: empty pores). Location of the slices is indicated in Figure 1.

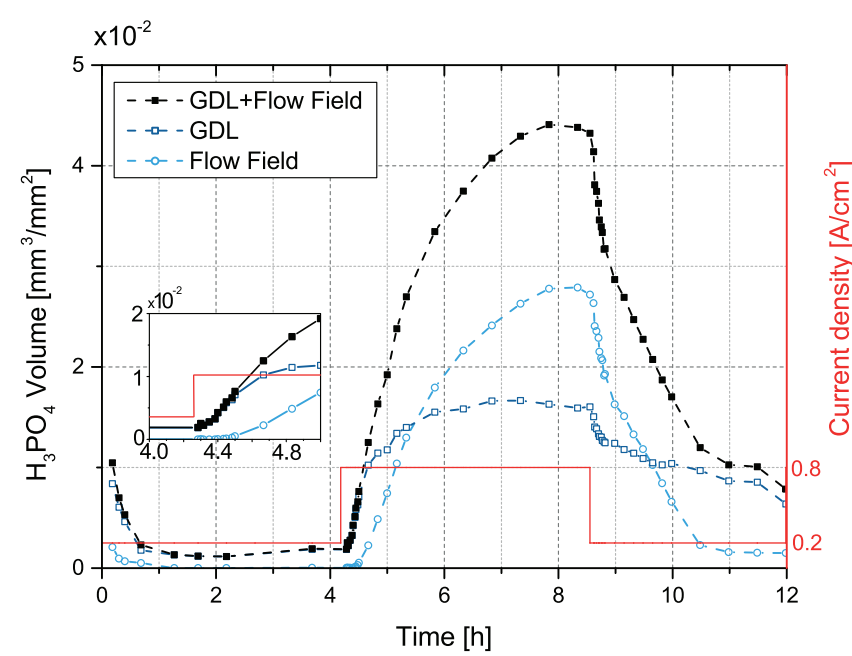

Figure 3. Volume of PA per active area in the GDL, flow field and total as a function of time in the slow cycling experiment (the dashed lines act as a guide for the eye).

with a decrease of PA volume in the flow field. After $10 \mathrm{~min}$, this sudden drop in PA saturation is followed by a slow and continuous decrease of electrolyte volume in the GDL until the end of the low current cycle after $12 \mathrm{~h}$. The volume of PA in the flow field decreases much faster than in the GDL and reaches a minimum after $3 \mathrm{~h}$ at $0.2 \mathrm{~A} \mathrm{~cm}^{-2}$. Interestingly the condition before the current step is not completely reached. Disconnected PA droplets sticking to the walls remain in the flow field, without continuous path these remainders are unable to diffuse back to the porous layers.

These droplets are ultimately lost from the electrolyte reservoir and lead to long term degradation due to electrolyte loss and possibly subsequent membrane and contact resistance increase.

A possible explanation for the observed strong migration of PA to the anode GDL and flow field channels can be given based on results and experience with phosphoric acid fuel cells. In these systems phosphoric acid is impregnated in a silicon carbide matrix. It has been described that, when the fuel cell is first started up or the current density is increased, electrochemical pumping is responsible for the migration of electrolyte from the cathode to the anode. ${ }^{21-23}$ This is due to the fact that the hydrogen phosphate anion has a finite, nonzero transport number. The transport number determines the fraction of current carried by each ion. When the hydrogen phosphate ion is carrying part of the current, a net movement of hydrogen phosphate from cathode to anode is induced where its charge gets balanced. Eventually a steady state is reached where no net movement of hydrogen phosphate can be observed anymore. At this point back diffusion (and/or hydraulic pressure) of phosphoric acid balances the migration process. In the PBI based systems only 2 PA molecules are bound per repeating unit of $\mathrm{PBI},{ }^{5}$ consequently $\mathrm{PA}$ also has a large mobility and a majority of the PA electrolyte is able to move freely within the

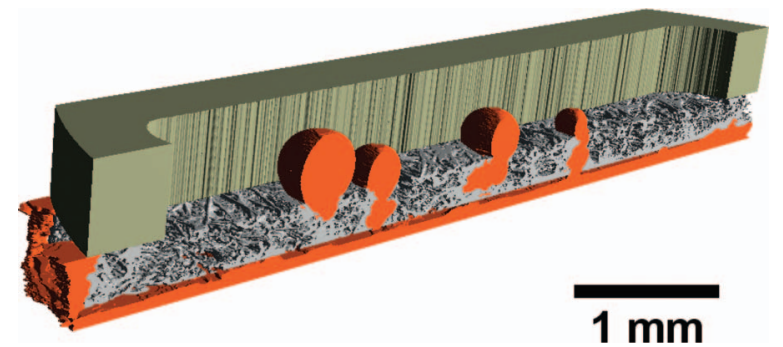

Figure 4. 3D rendering of a vertical along the flow field channel (orange: PA and catalyst layer; olive: flow field; gray: carbon fibers). 


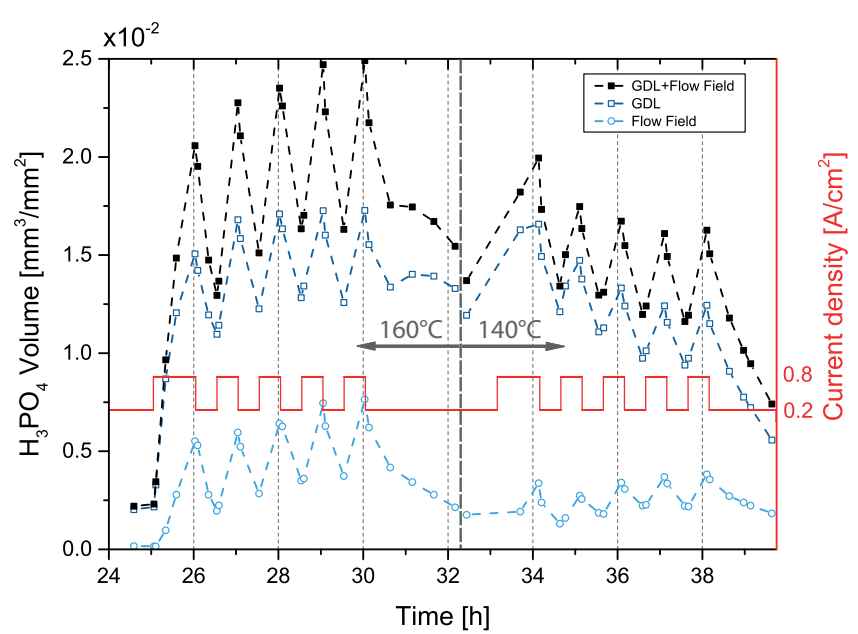

Figure 5. Volume of PA per active area in the GDL, flow field and total as a function of time in the fast cycling experiment for $160^{\circ} \mathrm{C}$ and $140^{\circ} \mathrm{C}$ (the dashed lines act as a guide for the eye).

molecular pores of the polymer backbone. A similar behavior as for PAFC can therefore also be expected for PBI based systems.

The dynamics of the PA migration and diffusion have been investigated using a fast cycling protocol. In Figure 5 the PA movement in the anode GDL and flow field is shown for five load cycles at $160^{\circ} \mathrm{C}$ and $140^{\circ} \mathrm{C}$. Essentially, a very similar electrolyte pumping effect can be observed also for the short current density cycles. Interestingly back diffusion of PA at $0.2 \mathrm{~A} \mathrm{~cm}^{-2}$ is slightly slower than migration at high current, resulting in an accumulation of electrolyte with each cycle. After about 3 cycles the maximum saturation within the GDL is reached. The flow field saturation on the other hand is continuously increasing but not to the same extent as in the slow cycling protocol.

At the end of the fifth cycle at $160^{\circ} \mathrm{C}$ a prolonged period of $0.2 \mathrm{~A} \mathrm{~cm}^{-2}$ was applied (the period from $30-33 \mathrm{~h}$ ). During this time a continuous decrease of PA in the flow field is observed while the saturation in the GDL, after an initial drop, stays about constant. This indicates a back diffusion of PA from the flow field through the macro pores of the GDL into the membrane similar to the slow cycling protocol.

At a reduced cell temperature of $140^{\circ} \mathrm{C}$ and the same cycling dynamics it becomes apparent that PA volume in the GDL is reduced during the first three cycles by about $30 \%$ as compared to $160^{\circ} \mathrm{C}$. A possible explanation for the reduced acid migration at lower temperatures can be rationalized as follows: A decrease in temperature leads to a decrease in PA concentration and hence to a net decrease in the dynamic viscosity of phosphoric acid. This in turn increases the molar proton conductivity ${ }^{24} \lambda^{+}$and hence reduces the electrolyte pumping effect toward the anode because a higher fraction of the current in the membrane is carried by protons.

In Figure 6 a comparison of the both cycling protocols is given with respect to electrolyte volume and saturation in the GDL. The maximum PA saturation of approximately $12 \%$ is reached for both experiments after $3 \mathrm{~h}$. With the applied cycling frequencies, load cycling obviously has no influence on the maximum saturation in the GDL, due to the initial imbalance between migration and diffusion as explained above.

In both experiments drainage of the GDL during extended times of low current density appears to be a two-step process as illustrated in Figure 6 for the slow cycling protocol. A closer look at the GDL in-plane saturation profiles (Figure 7) after stepping back to $0.2 \mathrm{~A}$ $\mathrm{cm}^{-2}$ reveals a bimodal saturation profile following the bimodal poresize distribution. Close to the flow field, accumulation of PA under the flow field ribs leads to the first saturation maximum. The second saturation peak $(675 \mu \mathrm{m})$ falls together with the porosity maximum of the GDL. At the beginning of PA drainage, after switching to low

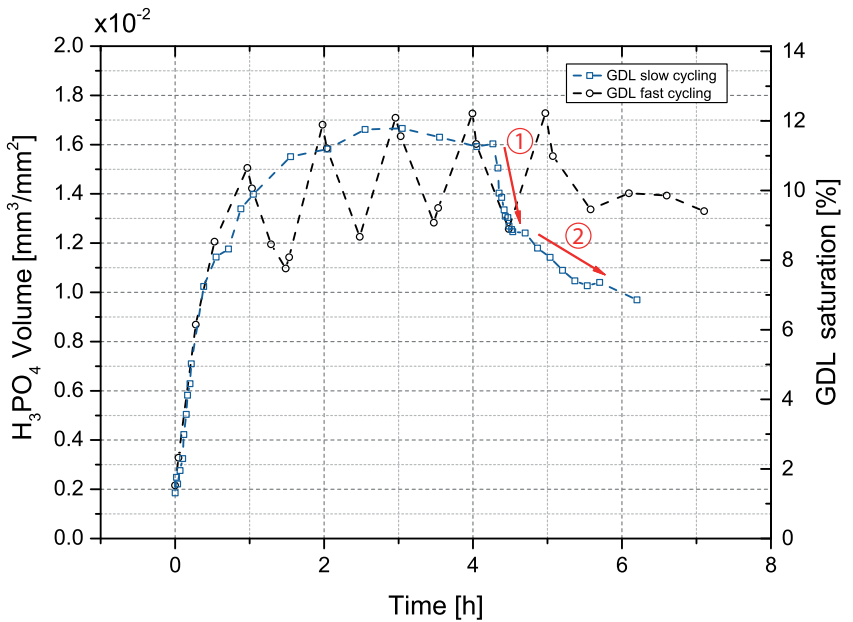

Figure 6. Comparison of the volume and saturation of PA in the GDL as a function of time (starting from $0.8 \mathrm{~A} \mathrm{~cm}^{-2}$ ) in the slow and fast cycling experiment at $160^{\circ} \mathrm{C}$ (the dashed lines act as a guide for the eye).

current, a fast decrease in saturation throughout the GDL can be observed (process 1 in Figure 6). Subsequently, the saturation close to the MPL reaches a minimum and further decrease in saturation is mainly driven by a decrease of PA under the ribs. The time constant for process (1) is smaller than for (2) and results in the observed two slopes in Figure 6. It is important to note that the PA saturation close to the MPL stays constant. This ensures a constant diffusion path for PA from the flow field back to the membrane.

In Figure 8 the mean grayscale value of each slice along the MEA thickness (z-coordinate, c.f. Figure 1) is plotted for a current jump from 0.2 to $0.8 \mathrm{~A} \mathrm{~cm}^{-2}$ in Figure $8 \mathrm{a}$ and for 0.8 to $0.2 \mathrm{~A} \mathrm{~cm}^{-2}$ in Figure $8 \mathrm{~b}$. The illustrated curves correspond to the times indicated in Figure 1. In this plot an increase in grayscale value is evidence of an increase in material for the specific slice. It can be clearly seen that with the increase in current density, the mean grayscale value in the anode and flow field increases due to the previously discussed electrolyte migration. The cathode GDL values slightly decrease, presumably due to migration of trace amounts (below the observable cluster size) of PA, further supporting the proposed acid migration process. Interestingly, a net shrinkage of the MEA by $16 \mu \mathrm{m}$ can be observed, although the volume of PA within the membrane is expected to grow due to increased water production at the cathode. ${ }^{13}$ Moreover, both catalyst layers as well as the membrane show an increase in grayscale value with a maximum for the anode electrode. One

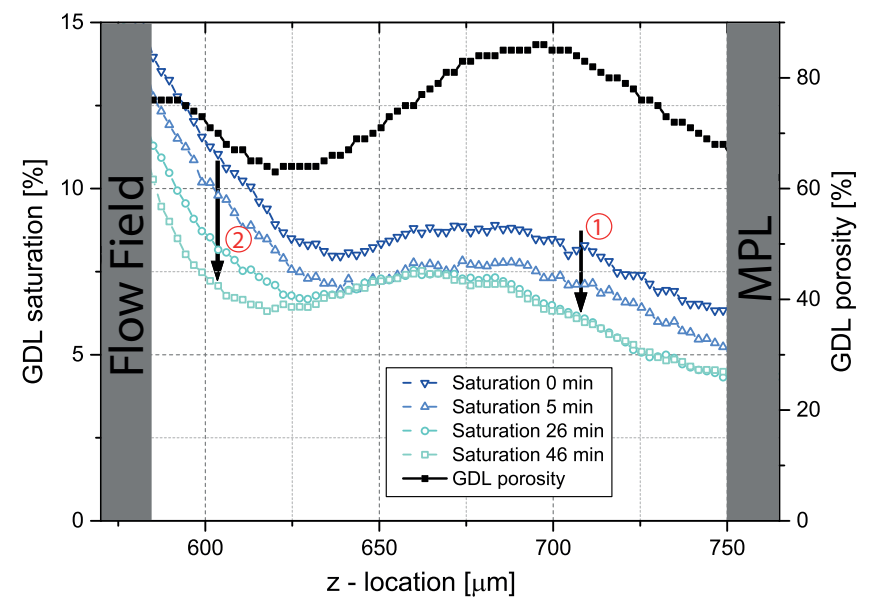

Figure 7. In-plane saturation and porosity profile of the GDL during drainage (the dashed lines act as a guide for the eye). 


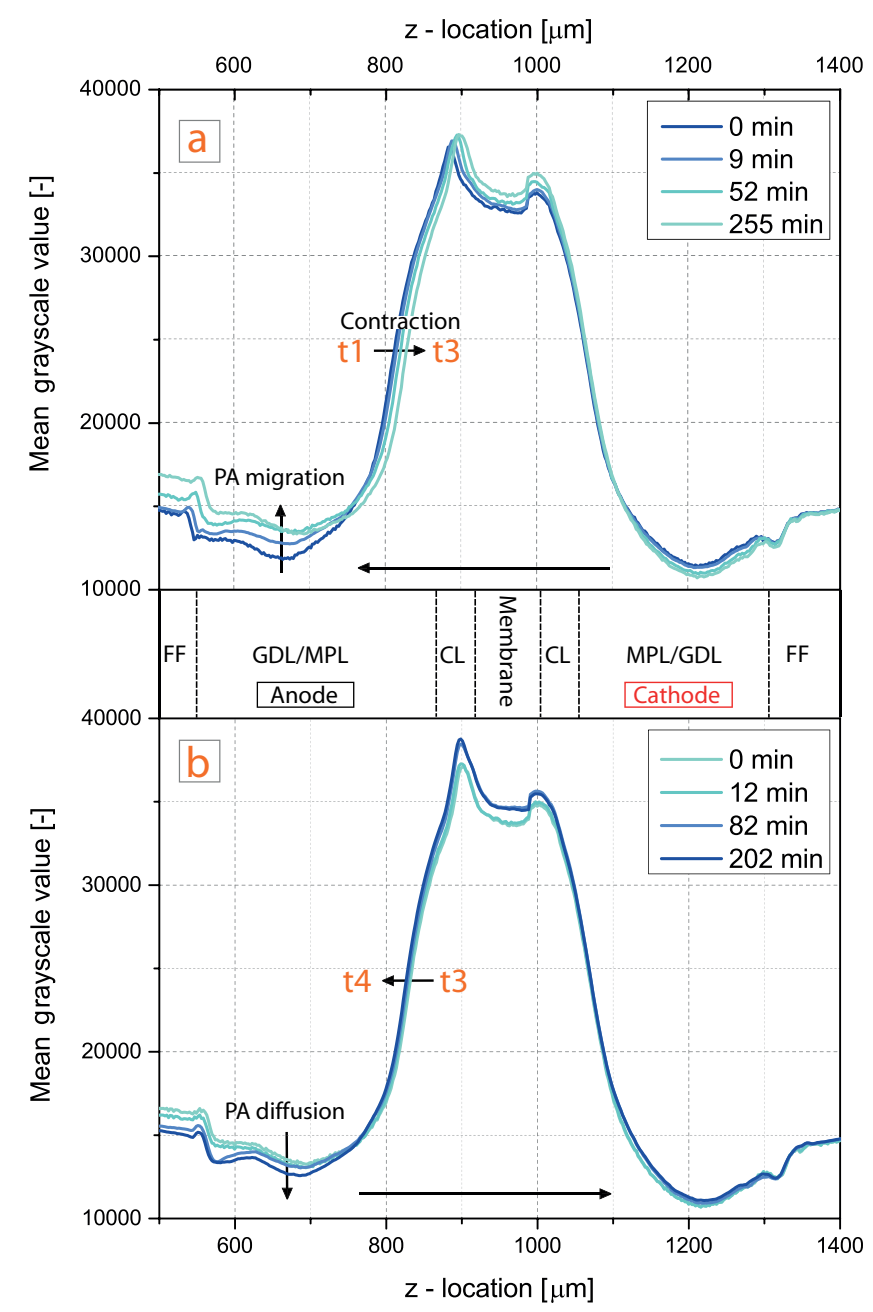

Figure 8. Mean grayscale distribution as a function of z-location (slice) for time step $\mathrm{t} 1 \rightarrow \mathrm{t} 3\left(0.8 \mathrm{~A} \mathrm{~cm}^{-2}\right)$ and $\mathrm{t} 3 \rightarrow \mathrm{t} 4\left(0.2 \mathrm{~A} \mathrm{~cm}^{-2}\right)$.

possible explanation is an increased degree of filling of previously empty micro-pores in the electrodes and molecular pores of the membrane caused by acid migration and water formation. In Figure $8 \mathrm{~b}$ the mean grayscale evolution during the subsequent current decrease is shown. The anode GDL exhibits a strong decrease in attenuation due to the back diffusion of PA from the GDL to the catalyst layer and membrane. This reverse process however does not cause an expansion of the MEA to the original thickness. However, additional increase in the maximum grayscale value of the electrodes and membrane can be observed, indicating that migrated PA primarily fills again previously unoccupied pore volume within electrodes and membrane. Clearly this process can only continue as long as there is void space available; the situation once full saturation is reached will be subject of future investigations.

PA quantification. - Information about the PA concentration will allow for retrieving important physical parameters, i.e. molar proton

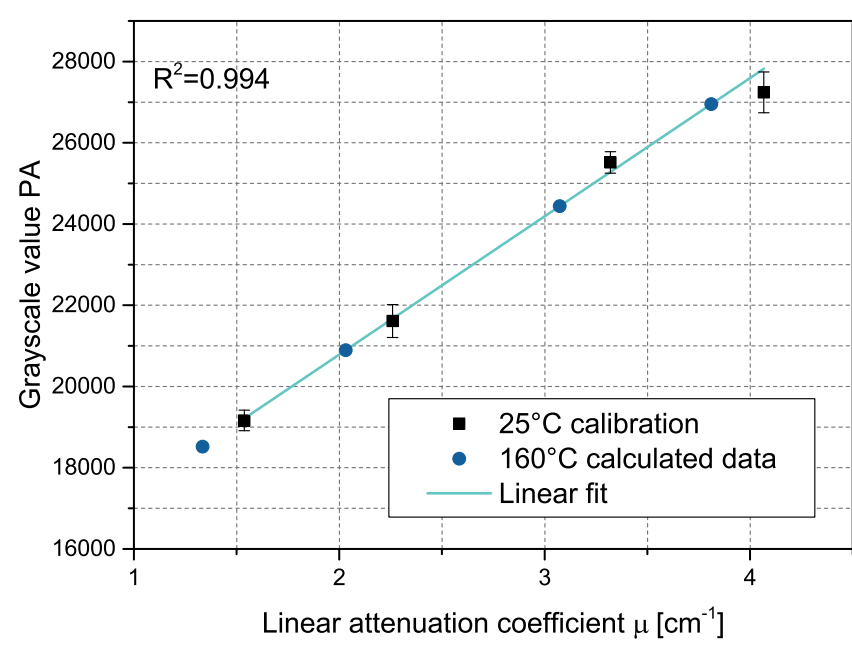

Figure 9. Measured grayscale value of phosphoric acid in the GDL at $25^{\circ} \mathrm{C}$ (black squares) and the corresponding linear attenuation coefficient. The values at $160^{\circ} \mathrm{C}$ (blue circles) are calculated using the linear fit of the $25^{\circ} \mathrm{C}$ data.

conductivity, oxygen diffusivity, etc. The methodology ${ }^{17}$ used in this work allows to obtain information about the local PA volume but also about the PA composition. In Table III literature data for mass attenuation and density is listed for 40, 60, 85 and $100 \mathrm{wt} \% \mathrm{H}_{3} \mathrm{PO}_{4}$. The product of mass attenuation and PA density establishes the linear relation to the grayscale values of the tomograms by means of a linear attenuation coefficient.

In Figure 9 this relation is plotted for the measured calibration samples at $23^{\circ} \mathrm{C}$ and the respective calculated grayscale values for $160^{\circ} \mathrm{C}$. The decrease in grayscale for higher temperatures results from the respective, known decrease in density. This calibration is used to determine the concentration of PA in the anode GDL. The values and results of the calculations are listed in Table IV. The in-operando PA concentration is $96.5 \pm 1.5 \mathrm{wt} \%$ and $95.5 \pm 1.5 \mathrm{wt} \%$ for $160^{\circ} \mathrm{C}$ and $140^{\circ} \mathrm{C}$, respectively, which is in good agreement with published data for pure $\mathrm{H}_{3} \mathrm{PO}_{4}$ vapor liquid equilibrium ${ }^{25}$ at low water partial pressure. No significant difference in the grayscale for PA at different current densities was observed. This is most probably due to the constant water vapor pressure in the differential cell set-up. Knowledge of the PA composition allows for quantification of migrated electrolyte during the current cycle experiments. For the slow cycling protocol, $2.8 \cdot 10^{-2} \mathrm{mg} \mathrm{mm}^{-2} \mathrm{H}_{3} \mathrm{PO}_{4}$ was found in the GDL and a total of $7.5 \cdot 10^{-2} \mathrm{mg} \mathrm{mm}^{-2}$ in the GDL and flow field at maximum saturation. This corresponds to approximately $23 \mathrm{wt} \%$ of the total electrolyte inventory of the cell. The rate with which the acid enters the GDL during the first 12 minutes after the increasing current step is $0.75 \mu \mathrm{g} \mathrm{min}^{-1} \mathrm{~mm}^{-2}$.

Practical implications of acid migration.- The observed acid migration has a range of implications with respect to fuel cell performance and durability. The consequences are discussed in this subsection.

The loss of electrolyte due to disconnected PA droplets within the GDL and the flow field channels during load cycling will inevitably lead to long term fuel cell degradation in the form of increasing

Table III. Results of the calibration experiment performed at $23^{\circ} \mathrm{C}$.

\begin{tabular}{|c|c|c|c|c|}
\hline Sample ID & $\begin{array}{c}\text { Mass attenuation } \\
\text { coefficient }^{29}\left[\mathrm{~cm}^{2} / \mathrm{g}\right]\end{array}$ & $\begin{array}{l}\text { Density }^{30} \\
{\left[\mathrm{~g} / \mathrm{cm}^{3}\right]}\end{array}$ & $\begin{array}{l}\text { Linear attenuation } \\
\text { coefficient }\left[\mathrm{cm}^{-1}\right]\end{array}$ & $\begin{array}{c}\text { Grayscale value } \\
{[-]}\end{array}$ \\
\hline $40 \mathrm{wt} \%$ & 1.348 & 1.141 & 1.537 & $19163 \pm 253$ \\
\hline $60 \mathrm{wt} \%$ & 1.632 & 1.384 & 2.259 & $21611 \pm 405$ \\
\hline $100 \mathrm{wt} \%$ & 2.174 & 1.871 & 4.067 & $27241 \pm 503$ \\
\hline
\end{tabular}


Table IV. Phosphoric acid composition as a function of grayscale value for the fast cycling experiment at $160^{\circ} \mathrm{C}$ and $140^{\circ} \mathrm{C}$.

$160^{\circ} \mathrm{C}$

\begin{tabular}{cccc}
\hline Sample ID & $\begin{array}{c}\text { Grayscale value } \\
{[-]}\end{array}$ & $\begin{array}{c}\text { Density } \\
{\left[\mathrm{g} / \mathrm{cm}^{3}\right]}\end{array}$ & $\begin{array}{c}\text { Linear attenuation } \\
\text { coefficient }\left[\mathrm{cm}^{-1}\right]\end{array}$ \\
\hline Fast cycling $160^{\circ} \mathrm{C}$ & $26097 \pm 273$ & 1.709 & $3.626 \pm 0.084$ \\
& & $140^{\circ} \mathrm{C}$ & \\
Fast cycling $140^{\circ} \mathrm{C}$ & $26109 \pm 273$ & 1.714 & $3.629 \pm 0.084$
\end{tabular}

membrane resistance. Reduced lifetimes and severe degradation of aged cells where the electrolyte inventory is already depleted due to PA evaporation can be expected.

The membrane thinning observed at high current density, was not recovered after switching back to low current density. Instead, an increase in the degree of pore filling of both electrodes and membrane was measured. Unlike low temperature perfluorosulfonic acid (PFSA) membranes, where membrane swelling is driven by strong osmotic pressure, the driving force in PBI based systems is primarily a concentration gradient. The driving force in HT-PEFCs is therefore much weaker and possibly not able to overcome the compression force in the cell. With membrane thinning, the long term degradation effects expected are consequently an increase in contact resistance if using a sealing concept based on constant gap. It requires further experiments to investigate the effects of repeated load cycling on membrane thinning and contact resistance.

The extensive migration of electrolyte is also assumed to possibly influence fuel cell performance under reformate operation. The PA saturation of GDL and catalyst layer on the anode can negatively influences fuel cell operation under reformate conditions because the reduced hydrogen partial pressure in reformate $\left(\mathrm{H}_{2}, \mathrm{CO}, \mathrm{CO}_{2}, \mathrm{H}_{2} \mathrm{O}\right.$ and $\mathrm{N}_{2}$ ) is more susceptible to mass transport limitations. Especially system concepts based on autothermal reforming and its low hydrogen partial pressure as compared to steam reforming, might be susceptible to performance losses.

The presented results should also serve as an input for the modelling community in order to understand the implications on fuel cell performance and degradation in more detail. Especially the processes taking place within the electrodes during and after current steps are critical for a successful long term operation. The results can also give new impulse for material design, i.e. pore size distribution of the MPL, catalyst layer and GDL.

Radiation damage.- LT-PEFCs are susceptible to X-ray radiation inducing performance degradation. ${ }^{26-28}$ In LT-PEFC few minutes of irradiation at the TOMCAT beamline lead to an observable decrease in performance. In order to investigate the radiation sensitivity of HTPEFC, the set-up was subjected for a period of $30 \mathrm{~min}$ uninterrupted irradiation at $20 \mathrm{keV} \mathrm{X}$-ray energy at the beamline. No voltage degradation could be observed. This fundamental difference to LT-PEFC is explained by the fact, that the membrane polymer, which is mainly degrading in LT-PEFC, is only a structural component in HT-PEFC which does not take part in the electrochemical processes. Polymer degradation and radiation induced changes in the wetting behavior of the GDL, however, can also for HT-PEFC not be excluded. Additional experiments would be necessary for quantifying possible impacts of radiation on fuel cell operation.

\section{Conclusions}

In this work synchrotron based X-ray tomographic microscopy (XTM) was used to image and quantify the phosphoric acid electrolyte redistribution in in-operando HT-PEFCs. Specifically the processes taking place under dynamic load conditions were investigated in order to identify potential fuel cell degradation mechanisms. It is shown that the examined PBI based membrane systems show extensive electrolyte migration from cathode to anode under high current density operation. This is attributed to the fact that negatively charged hydro- gen phosphate anions carry part of the ionic current. This is unlike low temperature polymer electrolyte fuel cells where the anions are covalently bound to the polymer backbone, while in HT-PEFC only a fraction of the phosphoric acid is chemically interacting with the polymer backbone. XTM for the first time revealed the current driven electrolyte migration in HT-PEFC.

It was demonstrated that the migration and consequently gas diffusion layer flooding of the anode is a relatively fast process, reaching steady state in the GDL after two and in the flow field after about four hours. The process seems not to be completely reversible with unconnected droplets of PA remaining in the flow field after returning to low current density. Furthermore, diffusion of PA, back from the GDL into the membrane and electrodes is a slower process and no steady state was reached after 8 hours.

Electrolyte migration is reduced when decreasing the cell temperature where the molar proton conductivity, and consequently the transport number of protons is higher. It was also observed that the MEA decreased in thickness by approximately $16 \mu \mathrm{m}$ after the first load cycle and did not regain its initial thickness afterwards. When the membrane has been compressed after a loss of PA it cannot regain thickness under the compressive load in the cell.

The grayscale values in the tomographic images were correlated to PA composition by means of calibration. Thus the amount of migrated electrolyte was determined from the volume and PA concentration. $23 \mathrm{wt} \%$ of the total $\mathrm{H}_{3} \mathrm{PO}_{4}$ inventory was found to migrate into the anode GDL and flow field at a current density of $0.8 \mathrm{~A} \mathrm{~cm}^{-2}$. Such intensive phosphoric acid migration has a range of potential consequences, which should be subject of further studies. First of all, long term degradation will occur due to loss of electrolyte with each load cycle due leaving unconnected droplets in GDL and flow field. Further with low hydrogen partial pressures in reformate operation the high PA saturation in the GDL could also have an influence on mass transport and consequently fuel cell performance. In case of complete stacks, a migration to adjacent cells might also be possible if the bipolar plates exhibit small defects, allowing PA to crossover. PAFC suffer from the same mechanism but intensive research has solved most of the problems with respect to acid migration, i.e. adjusting pore size distribution on anode and cathode and pressure increase on the anode.

\section{Acknowledgments}

Financial support by BASF SE, precise machining work by M. Hottiger, software and electronic support by T. Gloor and support at the TOMCAT beamline by J. Roth, J. Eller, A. Lamibrac, I. Mayrhuber and T. Lochner are gratefully acknowledged.

\section{References}

1. J. S. Wainright, J. Wang, D. Weng, and R. F. Savinell, and and M. Litt, J. Electrochem. Soc., L121 (1995).

2. T. J. Schmidt and J. Baurmeister, ECS Trans., 3, 861 (2006)

3. J. Spendelow, T. Nguyen, C. Houchins, E. Martin, and D. Papageorgopoulos, DOE Hydrogen Fuel Cells Program Record, 1 (2012).

4. L. Vilciauskas, M. E. Tuckerman, G. Bester, S. J. Paddison, and K. Kreuer, Nat. Chem., 4, 461 (2012).

5. Q. Li, R. He, R. W. Berg, A. H. Hjuler, and N. J. Bjerrum, Solid State Ionics, 168, 177 (2004).

6. Q. Li, J. O. Jensen, R. F. Savinell, and N. J. Bjerrum, Prog. Polym. Sci., 34, 449 (2009). 
7. J. Mader, L. Xiao, T. J. Schmidt, and B. C. Benicewicz, in Fuel Cells II, G. G. Scherer, Editor, vol. 216, p. 63, Springer, Berlin Heidelberg (2008).

8. C. Wannek, I. Konradi, J. Mergel, and W. Lehnert, Int. J. Hydrogen Energy, 34, 9479 (2009).

9. C. Hartnig and T. J. Schmidt, Electrochim. Acta, 56, 4237 (2011).

10. R. Makharia, M. F. Mathias, and D. R. Baker, J. Electrochem. Soc., 152, A970 (2005)

11. J. Eller, T. Rosén, F. Marone, M. Stampanoni, A. Wokaun, and F. N. Büchi, J. Electrochem. Soc., 158, B963 (2011).

12. R. Flückiger, F. Marone, M. Stampanoni, A. Wokaun, and F. N. Büchi, Electrochim. Acta, 56, 2254 (2011)

13. R. Kuhn, J. Scholta, P. Krüger, C. Hartnig, W. Lehnert, T. Arlt, and I. Manke, J. Power Sources, 196, 5231 (2011).

14. W. Maier, T. Arlt, C. Wannek, I. Manke, H. Riesemeier, P. Krüger, J. Scholta, W. Lehnert, J. Banhart, and D. Stolten, Electrochem. Commun., 12, 1436 (2010).

15. W. Maier, T. Arlt, K. Wippermann, C. Wannek, I. Manke, W. Lehnert, and D. Stolten, J. Electrochem. Soc., 159, F398 (2012).

16. P. Boillat, J. Biesdorf, P. Oberholzer, A. Kaestner, and T. J. Schmidt, J. Electrochem. Soc., 161, F192 (2014).

17. S. H. Eberhardt, F. Marone, M. Stampanoni, F. N. Büchi, and T. J. Schmidt, J. Synchrotron Radiat., 21, 1319 (2014).
18. J. Roth, J. Eller, F. Marone, and F. N. Büchi, J. Phys. Chem. C, 117, 25991 (2013)

19. M. Stampanoni, A. Groso, A. Isenegger, G. Mikuljan, Q. Chen, A. Bertrand, S. Henein, R. Betemps, U. Frommherz, and P. Böhler, et al. U. Bonse, Editor. Proc. SPIE, 6318, U199 (2006).

20. F. Marone and M. Stampanoni, J. Synchrotron Radiat., 19, 1029 (2012).

21. H. R. Kunz, Electrochem. Soc. Proc., 99-14, 191 (1999).

22. H. R. Kunz, ECS Trans., 11, 1447 (2007).

23. T. Murahashi, in Encyclopedia of Electrochemical Power Sources, C. K. Dyer, P. T. Moseley, Z. Ogumi, D. A. J. Rand, and B. Scrosati, Editors, p. 564 (2009).

24. C. Korte, in Fuel Cell Science and Engineering: Materials, Processes, Systems and Technology, D. Stolten and B. Emonts, Editors, p. 335, Wiley-VCH GmbH \& Co. KGaA, Weinheim, Germany (2012).

25. S. Lang, T. J. Kazdal, F. Kühl, and M. J. Hampe, J. Chem. Thermodyn., 68, 75 (2014).

26. J. Roth, J. Eller, and F. N. Büchi, J. Electrochem. Soc., 159, F449 (2012).

27. J. Eller and F. N. Büchi, J. Synchrotron Radiat., 21, 82 (2014).

28. J. Eller, J. Roth, F. Marone, M. Stampanoni, A. Wokaun, and F. N. Büchi, J. Power Sources, 245, 796 (2014).

29. http://physics.nist.gov/PhysRefData/FFast/html/form.html.

30. D. I. MacDonald and J. R. Boyack, J. Chem. Eng. Data, 14, 380 (1969). 